\title{
Transverse beam splitting made operational: Key features of the multiturn extraction at the CERN Proton Synchrotron
}

\author{
A. Huschauer, A. Blas, J. Borburgh, S. Damjanovic, S. Gilardoni, M. Giovannozzi, \\ M. Hourican, K. Kahle, G. Le Godec, O. Michels, and G. Sterbini \\ CERN, CH 1211 Geneva 23, Switzerland \\ C. Hernalsteens \\ Ion Beam Applications, BE 1348 Louvain-la-Neuve, Belgium \\ (Received 12 March 2017; published 14 June 2017)
}

\begin{abstract}
Following a successful commissioning period, the multiturn extraction (MTE) at the CERN Proton Synchrotron (PS) has been applied for the fixed-target physics programme at the Super Proton Synchrotron (SPS) since September 2015. This exceptional extraction technique was proposed to replace the longserving continuous transfer (CT) extraction, which has the drawback of inducing high activation in the ring. MTE exploits the principles of nonlinear beam dynamics to perform loss-free beam splitting in the horizontal phase space. Over multiple turns, the resulting beamlets are then transferred to the downstream accelerator. The operational deployment of MTE was rendered possible by the full understanding and mitigation of different hardware limitations and by redesigning the extraction trajectories and nonlinear optics, which was required due to the installation of a dummy septum to reduce the activation of the magnetic extraction septum. This paper focuses on these key features including the use of the transverse damper and the septum shadowing, which allowed a transition from the MTE study to a mature operational extraction scheme.
\end{abstract}

DOI: 10.1103/PhysRevAccelBeams.20.061001

\section{INTRODUCTION}

To provide high-intensity beams for fixed-target physics at the SPS, the longitudinal structure delivered by the PS has to comply with certain requirements. In order to reduce beam loading and to provide an almost continuous spill toward the experimental facilities, uniform filling of the SPS is desired. Considering that the length of the SPS is about eleven times the circumference of the PS, and that a gap for the rise time of the SPS kickers is needed, the nonresonant CT process was proposed in 1973 [1]. This extraction technique, which occurs over five turns at $14 \mathrm{GeV} / c$, allows to optimize the duty cycle as only two subsequent extractions from the PS are necessary to fill the SPS. On the downside, the CT extraction comes with the major drawback of significant beam loss occurring at multiple locations around the ring [2], leading to a high dose to personnel during accelerator maintenance and repair as well as too long cool down times.

Therefore, the MTE technique was proposed to replace the CT process in 2001 [3]. MTE is a resonant extraction

*alexander.huschauer@cern.ch

Published by the American Physical Society under the terms of the Creative Commons Attribution 4.0 International license. Further distribution of this work must maintain attribution to the author(s) and the published article's title, journal citation, and DOI. mechanism, which exploits advanced concepts of nonlinear beam dynamics and applies a fourth-order stable resonance to perform beam splitting in the horizontal phase space. The resulting beamlets-one core and four islands-are then extracted over five turns. More detailed information about the implementation of MTE in the PS and the theoretical treatment of adiabatic trapping can be found in [4,5].

Due to the complexity of the MTE scheme, its operational implementation has had to overcome many challenges. In 2010, about one month of operational experience could be gathered with this technique and two major issues were identified [6]: (i) significant fluctuations in the efficiency of the transverse splitting, in the losses at extraction and in the trajectories in the transfer lines; (ii) unacceptably high radioactive activation of the magnetic extraction septum (SMH16).

In order to overcome these problems, additional measures, which were not foreseen in the initial design of MTE [4], had to be implemented. This concerned the development of a so-called dummy septum (TPS15), i.e., a passive absorber to shield SMH16, and its installation in straight section (SS) 15 of the PS during the Long Shutdown 1 (LS1) between 2013 and 2014 [6]. A certain fraction of the losses during the extraction is due to the debunched longitudinal structure of the beam: during the rise time of the fast kickers, the debunched beam is swept from the internal to the external side of SMH16, causing unavoidable beam loss. Using TPS15, the activation of SMH16 can 


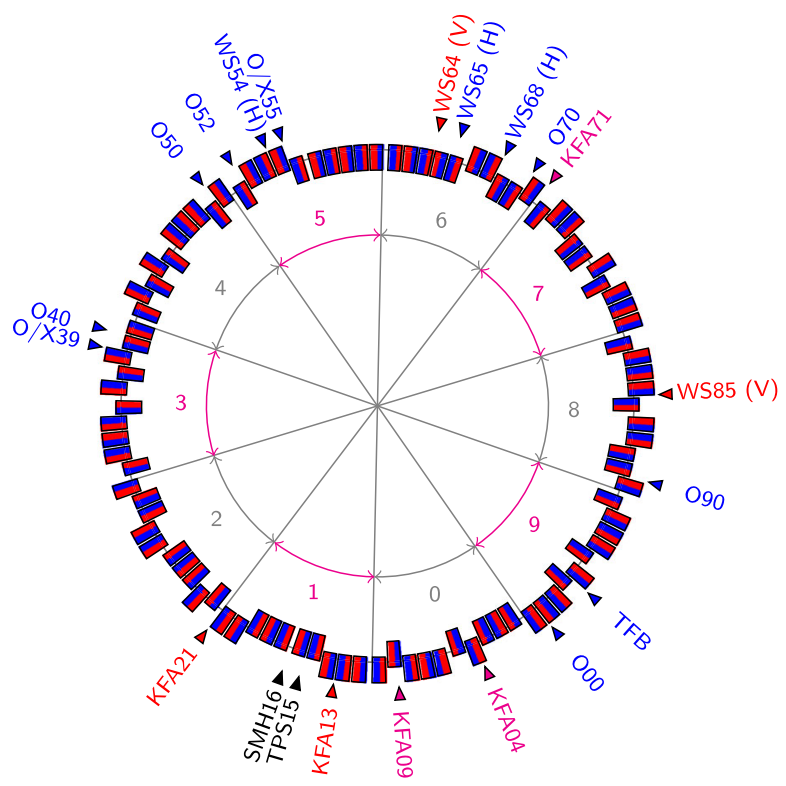

FIG. 1. Layout of the PS ring including the combined-function main magnets (red and blue rectangles for the focusing and defocusing parts, respectively) and the location of the key elements for MTE. The ten different sectors of the PS are indicated by the numbers and the radial offset between the rectangles accounts for the four different magnet types (see [7] for more detailed information).

be reduced by relocating these losses from SS16 to the well-shielded SS15. It was only after the installation of this device that the MTE commissioning could be resumed. A sketch of the PS layout is shown in Fig. 1, including the main elements for MTE, namely the dedicated sextupoles and octupoles to perform the transverse splitting (located in SS39 and SS55), the octupoles to correct nonlinear coupling (located in even SSs) and the different fast extraction kickers (KFAs). In addition, the location of the transverse damper (TFB) is indicated. The use of this device was not foreseen in the initial design, but during the MTE commissioning stages the beneficial impact of a transverse dipolar excitation on the splitting efficiency was realized. Horizontal and vertical beam wire scanners (WSs) are indicated as well.

In Fig. 2, the rf voltage programme of the main $10 \mathrm{MHz}$ accelerating cavities together with the time evolution of the strengths of the key elements for MTE is shown. The eight bunches arriving from the PS Booster (PSB) experience a longitudinal double splitting on an intermediate plateau and upon arrival on the flat top, the rf voltage is significantly reduced to minimize the coupling between the longitudinal and the transverse planes. Moreover, the second order chromaticities are desired to be small to further reduce the modulation of the horizontal tune and to assure that particles with different momenta cross the resonance $4 Q_{x}=25$, which is excited by the dedicated multipole magnets, almost simultaneously. The nonlinear elements
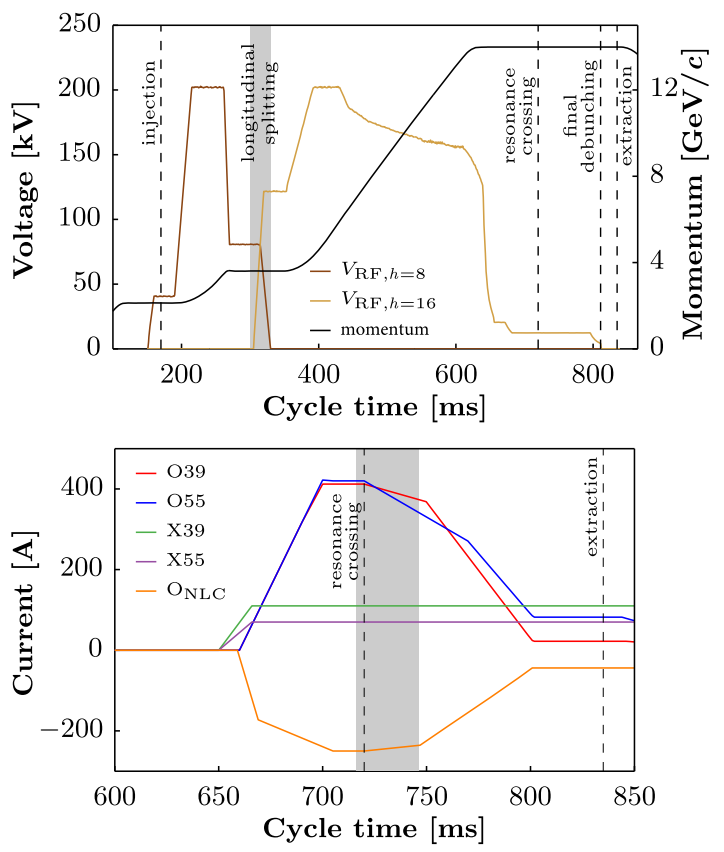

FIG. 2. Top: rf voltage program and magnetic cycle of the PS. Injection occurs at $2.14 \mathrm{Gev} / c$ and extraction at $14 \mathrm{GeV} / c$. Bottom: Operational functions of the octupoles and sextupoles applied to perform the transverse splitting. $\mathrm{O}_{\mathrm{NLC}}$ represents the family of octupoles dedicated to the correction of nonlinear coupling. The grey area corresponds to the time during which the transverse dipolar excitation is active.

induce negative detuning with amplitude and determine the size and the shape of the islands. Furthermore, the sextupoles are also used to reduce linear chromaticity. By means of additional quadrupoles, the horizontal tune is then programmed to cross the resonance and perform the horizontal splitting. At the end of the splitting process, the positions of the islands have to be rotated in the phase space in order to prepare the MTE beam for extraction in SS16. Subsequently, the beam is debunched and extracted towards the SPS.

In this paper, the key features of the MTE process are reviewed in detail by means of several experimental results and supporting simulation studies. These activities were essential to increase the understanding of MTE and eventually led to the implementation of appropriate measures to overcome the aforementioned problems. As a result of these studies, the MTE process was operationally deployed and has been used to deliver high-intensity beams to the SPS as of September 2015. Since then, it has successfully replaced the CT extraction. It is worth mentioning that a complete account of the performance analysis of MTE in the PSB, and the PS and SPS rings has been carried out and can be found in [8,9], respectively.

This paper addresses the aspects related to the transverse splitting and its fluctuations in Sec. II and then discusses the essential role of the transverse dipolar excitation (see Sec. III). The nonlinear optics at extraction, which accounts 
for the necessary rotation of the islands, is reviewed in Sec. IV, while the septum shadowing is considered in Sec. V. Finally, some conclusions are drawn in Sec. VI.

\section{ANALYSIS OF SPLITTING EFFICIENCY}

The efficiency of the transverse splitting is the natural figure-of-merit of the MTE performance and is defined as

$$
\eta_{\mathrm{MTE}}=\frac{\left\langle I_{\text {Island }}\right\rangle}{I_{\text {Total }}}
$$

where $\left\langle I_{\text {Island }}\right\rangle$ and $I_{\text {Total }}$ stand for the average intensity in each island and the total beam intensity, respectively. The nominal efficiency is 0.20 , corresponding to an equal beam sharing between islands and core. One of the challenges of working with transversely split beams is the fact that it is difficult to directly measure the locations of the various beamlets in the phase space. Therefore, $\eta_{\mathrm{MTE}}$ is inferred from phase space projections, which are obtained by using horizontal WSs or from beam current measurements in the transfer line after extraction of the five beamlets.

\section{A. Experimental observations}

One major show-stopper for the operational deployment of the MTE technique in 2010 was related to the observation of significant fluctuations of the splitting efficiency,
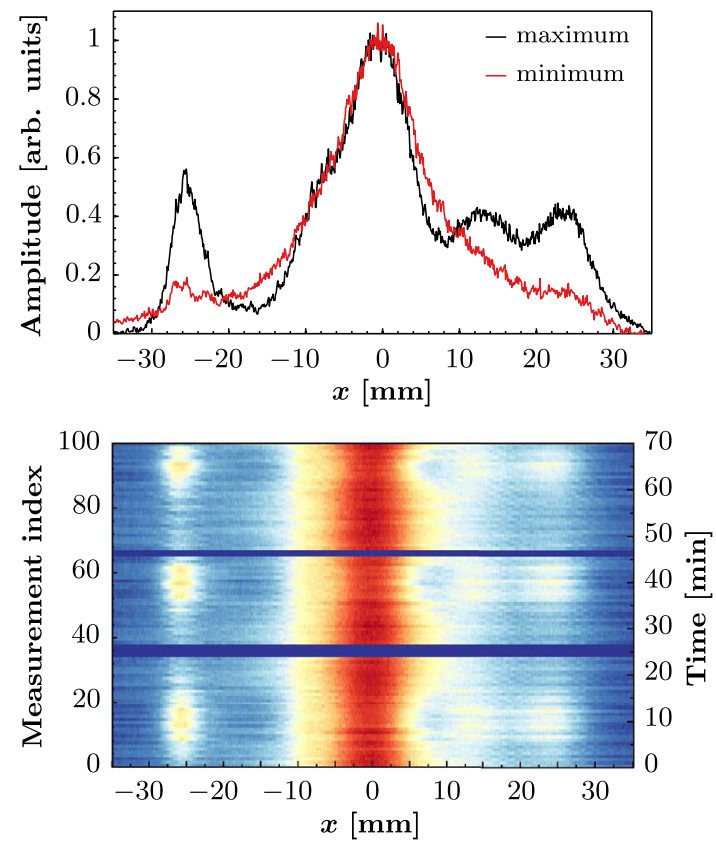

FIG. 3. Top: Comparison between good and bad splitting efficiency based on horizontal profiles measured with WS54. Bottom: Waterfall representation of multiple measured horizontal profiles, with the color scale corresponding to the amplitude of the data shown in the image at the top. Each measurement was recorded on a different cycle and clear oscillations of the beamlets' intensities are visible. which occasionally led to almost no population of the islands at all. After LS1 and the installation of TPS15, an extensive experimental campaign was started to identify the source of these fluctuations (see also [10] for more detailed information).

Figure 3 shows the evolution of several horizontal profile measurements with the WS in SS54 (WS54). The WS in this straight section of the PS was chosen, as the projection reveals rather distinct beamlets due to the orientation of the horizontal phase space. Clear oscillations of the intensities captured in the various beamlets are observed, with oscillation periods in the order of tens of minutes. This phenomenon caused cycle-by-cycle variations of $\eta_{\mathrm{MTE}}$ and significant deviations from the minimum acceptable value of 0.19 set by the SPS.

Subsequently, the stability of various parameters, which could perturb the splitting process, such as the horizontal tune or the rf voltage, was investigated on the MTE cycle; however, no clear correlation between these parameters and the measured fluctuations could be identified. Furthermore, the stability of the closed orbit was investigated and no oscillations were observed.

In the framework of the aforementioned investigations, an important observation was made when injecting argon ions into the PS, which occurs at approximately two-third of the rigidity of proton beams. Figure 4 shows a transverse tune measurement and, apart from the dominant horizontal
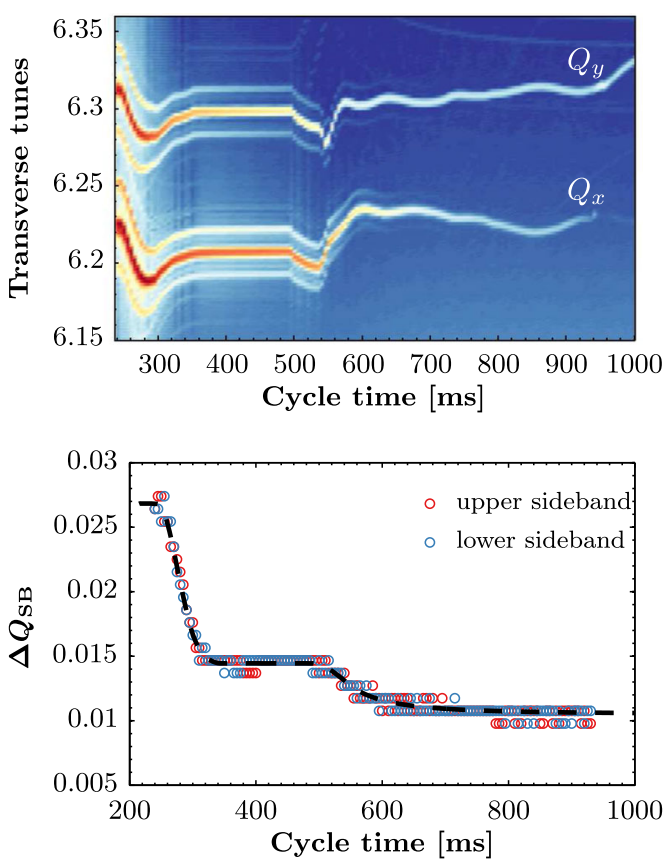

FIG. 4. Top: Tune measurement along an argon ion cycle. The average value over 900 cycles is shown. Sidebands are visible in both planes. Bottom: Distance of the sidebands from the horizontal tune. The dashed line corresponds to the expected distance of the sideband frequency $f_{\mathrm{SB}}=5 \mathrm{kHz}$ from the main tune peak along the cycle. 
and vertical tune lines, important sidebands are clearly visible. The frequency $f_{\mathrm{SB}}$ of these sidebands can be determined via the revolution frequency $f_{\text {rev }}$ and the distance to the main tune line $\Delta Q_{\mathrm{SB}}$

$$
f_{\mathrm{SB}}=\Delta Q_{\mathrm{SB}} \times f_{\text {rev }}
$$

and the analysis showed that $f_{\mathrm{SB}}$ corresponds to $5 \mathrm{kHz}$. The next step was to understand whether this observation could be explained by an actual modulation of the magnetic field at $5 \mathrm{kHz}$, which would then be directly transmitted to the beam, or if the tune measurement system itself was subject to an external disturbance.

To measure the magnetic field in the PS, pick-up coils are installed inside the reference main magnet outside the tunnel. The provided signals actually correspond to the time derivative of the magnetic field $\dot{B}=d B / d t$, and are used for longitudinal beam control as well as for the regulation of the power supplies [11].

In Fig. 5, measurements of the $\dot{B}$-signal on the argon cycle are depicted. An analysis of the frequency spectrum revealed an important component at $5 \mathrm{kHz}$ and, therefore, it was concluded that this oscillation is indeed present on the magnetic field. More interestingly, the amplitude of the peak at $5 \mathrm{kHz}$ varied over time and was observed to change at the same rate as the amplitude of the previously discussed sidebands of the measured tune.
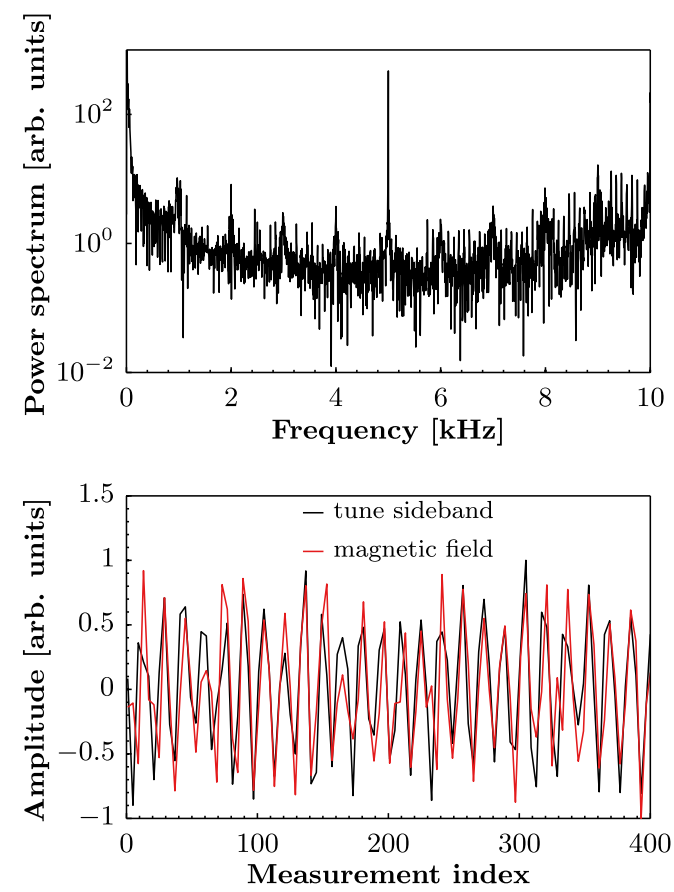

FIG. 5. Top: The FFT of $\dot{B}$ measured on an argon cycle reveals an important peak at $5 \mathrm{kHz}$. Bottom: The amplitude of the peak at $5 \mathrm{kHz}$ shown in the top figure oscillates in the same way as the amplitude of the sideband of the horizontal tune discussed in Fig. 4.
Subsequently, it was realized that $5 \mathrm{kHz}$ is equal to the frequency of the switch-mode power converters (PCs) used to power the different circuits of the pole-face windings (PFWs), which are special auxiliary windings installed inside the combined function main units (MUs) and are used to control transverse tunes and linear chromaticities [12]. This fact together with the result of the aforementioned magnetic measurements strengthened the suspicion of the fluctuations being related to the PFWs.

Therefore, local measurements of the current, i.e., directly at the exit of the PC, were conducted, while the horizontal profiles were recorded with WS54. A peak-topeak current ripple of $120 \mathrm{~mA}$ at $5 \mathrm{kHz}$ was observed on two out of five circuits, which corresponds to $20 \times 10^{-4}$ times the operational value and was clearly exceeding the specification of $\sim 1 \times 10^{-4}$ [13]. In Fig. 6, the oscillation of the intensity of the outermost island obtained with WS54 is compared to the variation of the amplitude of the spectral line at $5 \mathrm{kHz}$ measured on the PFWs, and very good agreement of the oscillation periods can be concluded.

All the observations presented in this section led to the understanding that the PFWs were at the origin of the intensity modulation of the islands. Furthermore, it was understood that different clocks, which are not synchronized with each other, are used to control the switching of the PCs. Any minor difference in phase and frequency between the clocks will, therefore, inevitably be transmitted to the current at the output of the PC. Due to their proximity inside the main units, the different circuits are magnetically coupled and the interference of the signals results in the modulation of the tune at $5 \mathrm{kHz}$ with varying amplitude in time.

Following a hardware intervention to reduce the current ripple at $5 \mathrm{kHz}$, the fluctuation of $\eta_{\mathrm{MTE}}$ was significantly improved. This achievement, which was the prerequisite to resume the transfer of MTE beams to the SPS, is also visualized in Fig. 7, where the intensity of the different beamlets measured with WS54 is shown to be constant in time.

In addition to the aforementioned investigations, which identified a modulation of the tune as the source of

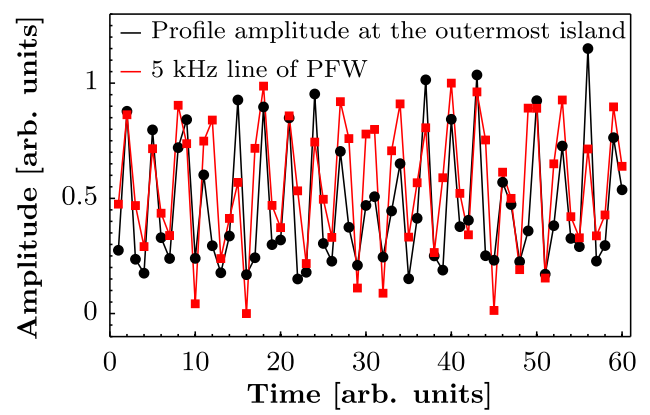

FIG. 6. Comparison between the time evolutions of the measured profile amplitude of the outermost island and the amplitude of the $5 \mathrm{kHz}$ current ripple of the PFWs. 


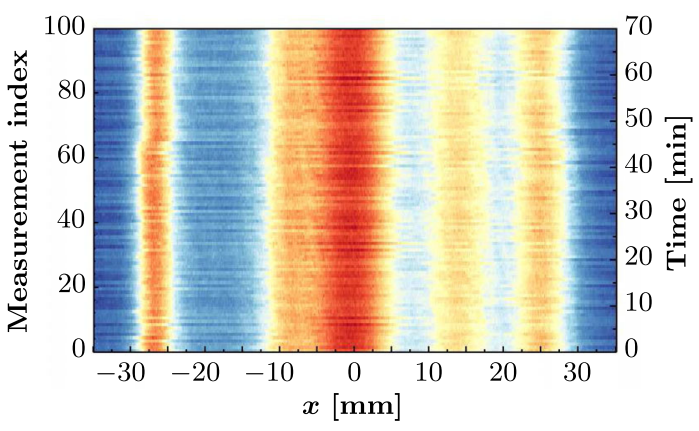

FIG. 7. Waterfall representation of multiple measured horizontal profiles after an intervention aimed at damping the current ripple of the PFWs at $5 \mathrm{kHz}$. Significantly improved stability with respect to the measurements shown in Fig. 3 is clearly visible.

cycle-by-cycle fluctuations of $\eta_{\mathrm{MTE}}$, the dependency of $\eta_{\text {MTE }}$ on a constant offset of the tune with respect to the nominal settings was studied. To address this point dedicated measurements were performed by introducing an offset in the settings of the quadrupoles used to control the horizontal and vertical tunes. Using the recorded values of the horizontal tune and $\eta_{\mathrm{MTE}}$, the two-dimensional probability distribution function has been estimated and the results are shown in Fig. 8.

The MTE efficiency features a rather symmetrical decrease around the nominal value of the horizontal tune, corresponding to $\Delta Q_{H}=0$. Moreover, it is possible to estimate that a reduction of about $5 \%$ of $\eta_{\mathrm{MTE}, \max }$ is obtained for a change of $\Delta Q_{H} \approx 0.5 \times 10^{-3}$, which can be considered the estimated threshold value in tune change that induces a non-negligible change of the MTE efficiency.

\section{B. Benchmarking of simulations}

The establishment of reproducible conditions for the horizontal splitting was an important milestone to continue the experimental studies with the MTE beam. Furthermore, it allowed to benchmark the results of simulations with the polymorphic tracking code (PTC) [14] with measurements

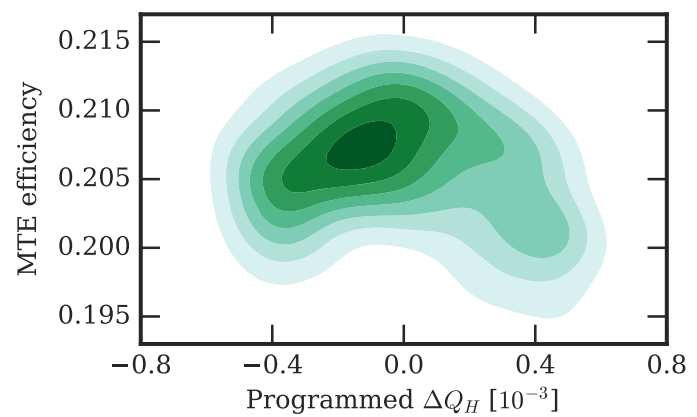

FIG. 8. Reconstructed two-dimensional probability distribution function for $\eta_{\text {MTE }}$ and programmed tune offset with respect to the nominal settings. A parabolic shape of the level lines of the probability distribution function is clearly visible. conducted with the WSs. This was essential, as the overall complexity of MTE requires an accurate and predictive model of the accelerator to further advance the understanding of this complex technique and, in particular, to understand the observations analysed in the previous section.

In order to obtain meaningful simulation results the description of the PS lattice is relying on an effective magnetic model. Two thin multipoles are inserted into each half unit (focusing or defocusing) of the MUs and these elements are then used to account for the multipolar components of the lattice, which are introduced by the MUs themselves and the auxiliary circuits. Based on chromaticity measurement on the flat top of the MTE cycle, quadrupolar, sextupolar and octupolar components are inferred and the corresponding kicks are distributed along the lattice.

In Fig. 9, a measured horizontal profile using WS54 is shown together with the corresponding phase space obtained by PTC simulations. When these measurements were recorded, several parameters, such as the horizontal tune and the strength of the transverse damper, were not optimized, leading to an important population of the core in the order of about $40 \%$ and, therefore, this example was selected for the purpose of illustration only. Already from this comparison between the simulated phase space and its

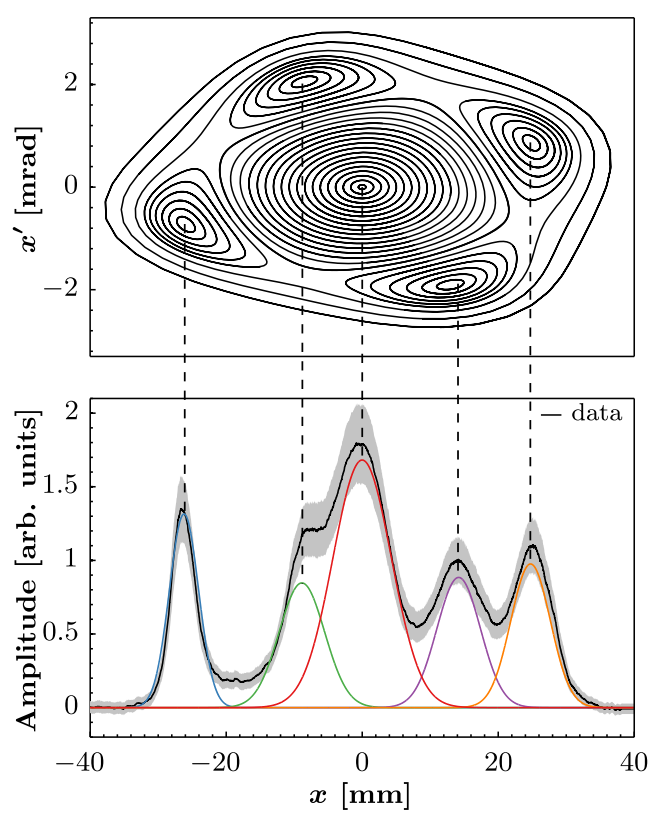

FIG. 9. Simulated horizontal phase space at the location of WS54 (top) and measured profile (bottom), which corresponds to an average value over 100 measurements. The grey band represents the one sigma standard deviation and the colored lines the Gaussian fits for the different beamlets, with the only constraint being the equal integral of the functions corresponding to the four islands. Excellent agreement between the predicted positions of the SFP and the actual measurement is obtained, which is indicated by the dashed lines. 
measured projection, very good agreement of the location of the stable fixed points (SFPs) between the model and reality can be concluded.

Figure 10 depicts the evolution of the horizontal profiles during the splitting process, which is composed of seventy different measurements. For this specific setup of the nonlinear elements, the main part of the splitting process occurs within $30 \mathrm{~ms}$ after crossing the resonance. Toward the end of the cycle, the rotation of the islands in the phase space, which is required to properly position the beamlets in the extraction region, is visible. During the entire splitting process, the locations of the five SFPs obtained by simulations are indicated by the colored markers on top of the measurement results. Even though the measurements were not performed with statistical significance, as every wire scan could only be carried out once within the available time frame of one hour, the smooth evolution of the data indicates an extremely reproducible splitting process. Minor discrepancies between measurements and simulations can be observed at the start of the rotation, i.e., around a time of $810 \mathrm{~ms}$ : this is due to the fact that the measurement of the MTE beam with a WS at this moment of the cycle actually takes about $5 \mathrm{~ms}$ and, accordingly, a time stamp of $810 \mathrm{~ms}$ corresponds to a time frame of $810 \pm 2.5 \mathrm{~ms}$. The agreement between measurements and simulation results validated the ability of the model to forecast the positions of the islands.

\section{Simulation studies}

The possibility of conducting 6D simulations with timevarying magnet strengths with the PyORBIT code [15] using PTC as underlying tracking code-enables the exploration of the extremely wide MTE parameter space. Based on various past simulation studies and, more importantly, on operational experience with the MTE

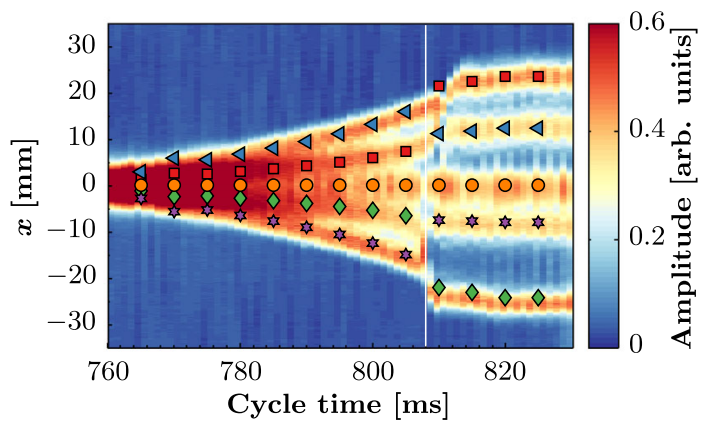

FIG. 10. WS54 measurements of the evolution of the transverse splitting along the cycle, with the simulated positions of the core (circle) and the islands indicated by the respective markers. In this case, the resonance was crossed at $760 \mathrm{~ms}$ and the rotation of the phase space started around $808 \mathrm{~ms}$ (indicated by the white solid line). Note the apparent discontinuity in the islands' positions at this moment, which is due to the projection effect from phase space to horizontal space. technique, the sensitivity of the process to variations of the horizontal tune is well known. This becomes also apparent in Fig. 10, where a variation of $\Delta Q_{x}=5 \times 10^{-3}$ leads to a separation between the core and the islands of about $25 \mathrm{~mm}$ (see also [8] for more detailed information about the settings of the magnetic elements applied during the splitting process).

In order to investigate the effect of a tune modulation at $5 \mathrm{kHz}$ on the efficiency of the transverse splitting, the dedicated multipole elements required to perform the horizontal splitting, i.e., sextupole and octupole magnets, were programmed according to the operationally used values and the horizontal tune was constantly increased over $50 \times 10^{3}$ turns to cross the resonance and perform the transverse splitting. The emittances of the transverse Gaussian particle distributions used in the simulations were based on measurements with WSs, and amounted to ( $1 \sigma$, normalized): $\varepsilon_{x}^{n}=12 \mathrm{~mm}$ mrad, $\varepsilon_{y}^{n}=5 \mathrm{~mm}$ mrad. The longitudinal particle coordinates were based on a measured reconstruction of the longitudinal phase space using tomography [16], with $\Delta p / p_{(1 \sigma)}=5 \times 10^{-4}$.

In addition, a $5 \mathrm{kHz}$ tune modulation corresponding to the maximum measured ripple amplitude of the PFWs circuits $(120 \mathrm{~mA})$ was included in the simulations. In Fig. 11, the resulting topology of the horizontal phase space
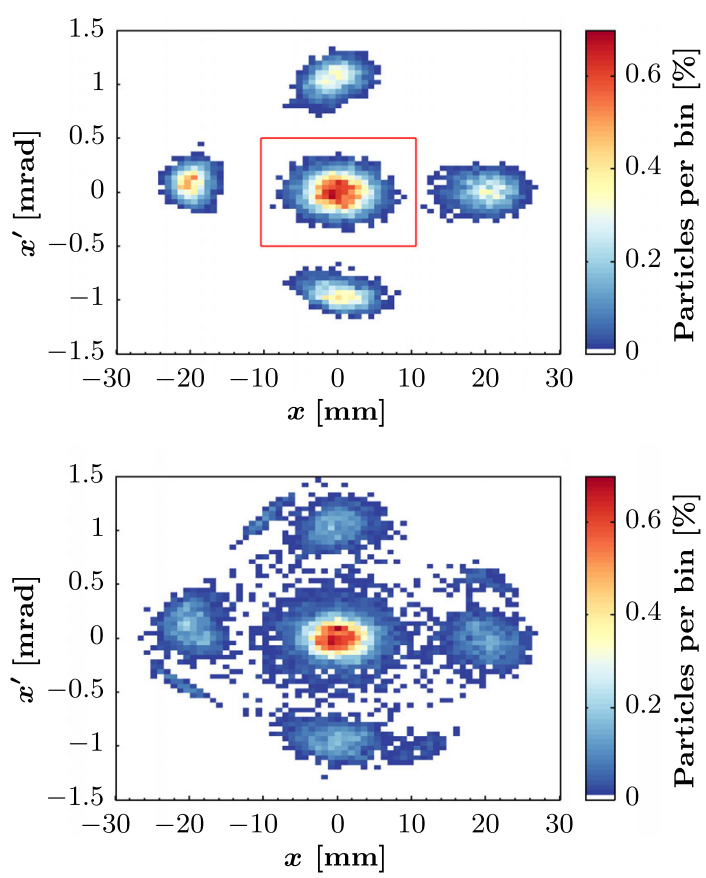

FIG. 11. Simulated horizontal phase space portraits in SS01 at the end of the splitting process in the absence of a tune ripple (top) and with a ripple amplitude corresponding to the maximum measured current ripple on the PFWs circuits (bottom). To quantify the effect on $\eta_{\mathrm{MTE}}$, particles inside the red boundary (drawn at $\pm 3.5 \sigma_{\text {core }}$ in $x$ and $x^{\prime}$ ) are considered core particles. The efficiency of the splitting process is clearly reduced by the presence of a tune ripple. 
is compared to the case without any tune ripple. Simulations without any ripple show an efficiency of only 0.145 , which is in agreement with experimental observations in the absence of the transverse damper. This device is operationally used to horizontally excite the beam for about $14 \times 10^{3}$ turns (the PS revolution period corresponds to $2.1 \mu \mathrm{s}$ ) after the crossing of the fourth order resonance to improve the trapping probability into the stable islands (see also Sec. III).

However, similar experimental results of $\eta_{\mathrm{MTE}}$ were achieved without using the transverse damper (see also Fig. 13), which highlights the very good capabilities of the effective nonlinear model for MTE. Furthermore, the tune ripple was found to severely affect the splitting process, leading to a reduction of $\eta_{\mathrm{MTE}}$ by more than $1 \%$ for the chosen parameters.

These simulation results clearly confirm what was expected based on experimental data: a tune ripple, if occurring at sufficiently large amplitude, is capable of preventing a proper splitting process. Following this conclusion, Fig. 12 depicts additional simulation results, which describe the dependency of the $\eta_{\mathrm{MTE}}$ on the amplitude and frequency of the ripple. These studies revealed that the splitting efficiency is even more severely affected by frequencies lower than $5 \mathrm{kHz}$. For higher frequencies, the impact on the splitting was found to be less important.
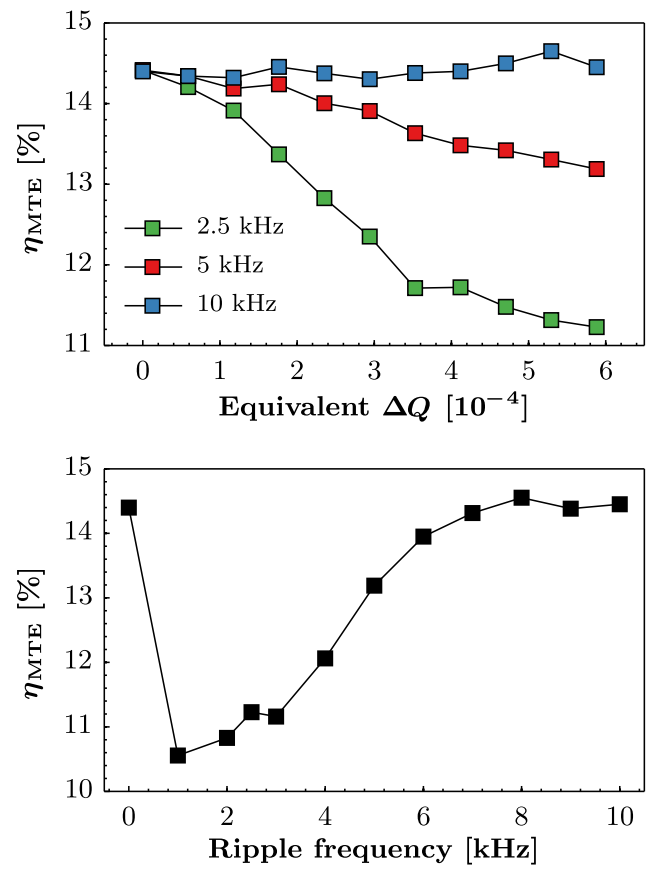

FIG. 12. Dependency of the splitting efficiency on the amplitude (top) and frequency (bottom) of the external perturbation. The ripple amplitude is expressed in terms of the amplitude of the sinusoidal perturbation of the horizontal tune. A low-frequency ripple significantly perturbs the splitting process.
In the case of conventional beam production, where the linear motion is of principal importance, the betatronic frequencies of the particles are considerably higher than several kHz. Considering, however, the betatronic motion around the SFPs in the islands, the situation changes as the oscillation frequencies slow down significantly. Investigations were conducted to determine the secondary oscillation frequencies of particles close to the islands' centers and it was understood that they indeed oscillate at frequencies in the low-kHz regime. Simulation studies with PTC revealed that the main oscillation frequency of a particle close to the SFP is around $2.5 \mathrm{kHz}$ and, in addition, the spectrum of the particle motion contains several higher harmonics due to the highly nonlinear motion. This is in agreement with the time-dependent simulation results discussed in Fig. 12, where a tune ripple at $2.5 \mathrm{kHz}$ was found to be especially detrimental to the transverse splitting.

Therefore, it was concluded that the depopulation of the islands in the presence of a low-frequency tune ripple occurs due to an overlap of the external excitation frequency with the particles' natural frequency of motion during the splitting process.

\section{TRANSVERSE DIPOLAR EXCITATION}

Since the early stages of the experimental activities carried out to study and implement MTE, it turned out that $\eta_{\mathrm{MTE}}$ had typical values lower than the target of 0.2. However, it was also observed that the use of a horizontal dipolar excitation during the resonance-crossing stage was increasing the value of the MTE efficiency up to the target or even beyond it. The mechanism leading to this observation is still a topic of intense theoretical studies [17].

The excitation is generated by means of the PS transverse damper. This device is based on a stripline kicker that normally works in closed loop with a transverse pick-up to preserve the beam emittance by damping injection oscillations and curing transverse instabilities. For the specific application of transverse beam splitting, the kicker is operated in open loop and provides a simple dipolar excitation. The main hardware parameters of the kicker are reported in [18].

The typical transverse displacement provided by the device is of the order of few tens of micron. Hence, this seems to indicate that the effect introduced by the transverse excitation is rather a resonant one than a possible modification of the transverse beam distribution. Another interesting observation is that the use of the dipolar excitation allowed reducing the dependence of $\eta_{\text {MTE }}$ on the value of the horizontal emittance before crossing the resonance.

The effect of the transverse damper on the beam during the splitting process can be described by an excitation amplitude and an excitation frequency, the latter being 
described by converting the frequency into fractional tune values. It is worth mentioning that the excitation can be switched on, in a single turn, at a given time and kept for a certain duration after which it is switched off, also in a single turn. Furthermore, thanks to recent upgrades, it is now also possible to generate an excitation with a timedependent frequency and, in this case, the initial frequency value and the frequency change per time, i.e., the slope of the function, can be accurately controlled. The impact of all these parameters on the beam dynamics has been carefully studied with dedicated beam measurements. A single, selected control parameter was randomly changed in a given interval and the corresponding value of $\eta_{\mathrm{MTE}}$ was recorded. The typical time span of each measurement session was of several hours, thus exceeding also the typical time scales of the variations of the MTE efficiency.

The results of the scans of the amplitude and frequency are shown in Fig. 13. For these cases the excitation frequency was kept constant in time. Each measured point is labeled with the level of beam losses at extraction measured on the beam loss monitor (BLM) in SS16.

A clear saturation of the effect of the damper excitation amplitude is visible, with a tendency of reducing $\eta_{\mathrm{MTE}}$ for an excitation close to the maximum amplitude of 1.0. Moreover, a sharp peak is observed in the scan over the excitation frequency. It is interesting to note that the excitation corresponding to the peak of $\eta_{\mathrm{MTE}}$ occurs at 0.2514 , which is a little larger than the resonant tune value.
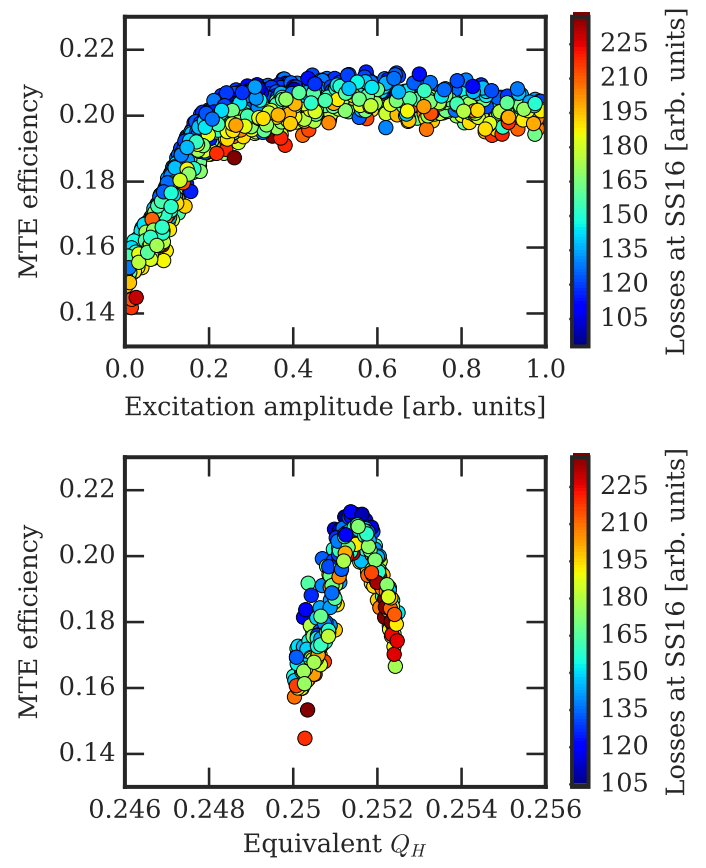

FIG. 13. Dependence of $\eta_{\text {MTE }}$ on the amplitude (top) and frequency (bottom) of the excitation generated by the transverse damper. The color scale provides the information on the extraction losses as measured by the BLM in SS16.
In both cases one can notice that the largest MTE efficiencies are correlated with low losses at extraction. The results of the frequency scan shown here refer to the scan over the fractional part of the equivalent tune value. Nonetheless, it is also possible to vary the integer part: accurate measurements showed, however, almost no dependence of the MTE efficiency on the integer part of the equivalent tune value.

It is also worth pointing out that the measurements show a value of $\eta_{\text {MTE }} \approx 0.15$ in the absence of excitation. Such a value is very close to that obtained with numerical simulations (see Fig. 12), i.e., 0.145, thus indicating the excellent predictivity of the model used for simulations.

The outcome of the scans over the start of the excitation and its duration is shown in Fig. 14. The parameter of the scan is in fact represented as difference with respect to the value used in normal operation. Strikingly, $\eta_{\mathrm{MTE}}$ appears to increase with decreasing length of the excitation window (Fig. 14, bottom). This phenomenon is due to the limited reproducibility of the quadrupolar component of the main field, which is also reflected in the larger spread of $\eta_{\mathrm{MTE}}$. Further improvement of the magnetic reproducibility of the machine will therefore be required to shorten the excitation window.

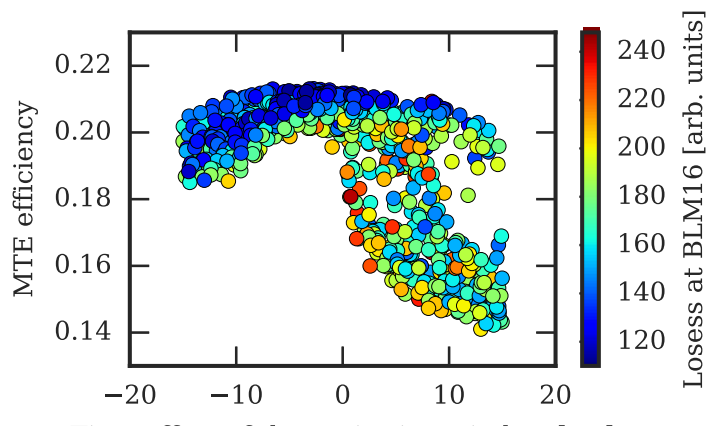

Time offset of the excitation window [ms]

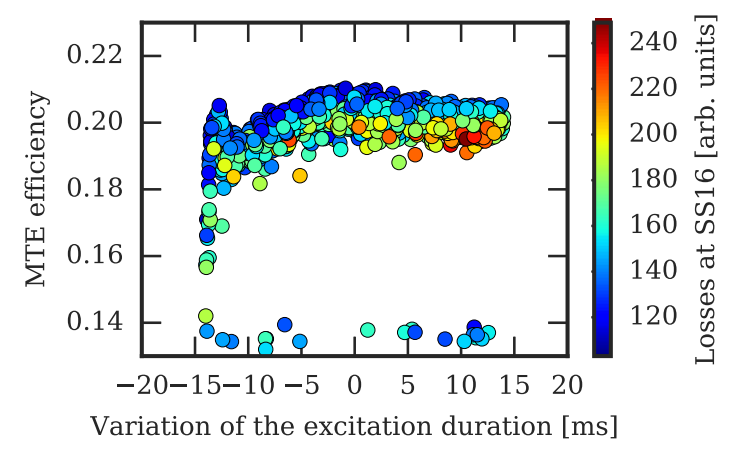

FIG. 14. Dependence of $\eta_{\text {MTE }}$ on the timing (top) and duration (bottom) of the excitation generated by the transverse damper. In both cases the scanned quantities are provided with respect to the nominal value used for MTE operation. The color scale provides the information on the extraction losses as measured by the BLM in SS16. 
In general, $\eta_{\mathrm{MTE}}$ features very broad and mild optima and the operational values of the parameters were already in the middle of the optimal ranges. Nevertheless, the beginning of the excitation window was advanced by $3 \mathrm{~ms}$ to move away from the sharp decrease of the MTE efficiency. Also in this case the higher $\eta_{\mathrm{MTE}}$ the lower are the measured losses at extraction.

The last study focused on the determination of the optimal parameters of the time-dependent excitation frequency, namely initial frequency and slope. The results are shown in Fig. 15.

A clear optimum is observed for the slope, corresponding to $\dot{Q}_{H} \approx 0.1 \mathrm{~s}^{-1}$. It is worth mentioning that the programmed tune change generated by the special tuning quadrupoles corresponds to about the same value of $\dot{Q}_{H}$. The scan over the initial value of the excitation frequency, which was conducted using the previously determined optimum slope, shows a relatively broad peak, with a position that is compatible with the optimum value of the frequency as obtained from the first scan (see Fig. 13, bottom). The broader peak with respect to the results presented in Fig. 13 (bottom) is a convenient feature as it contributes to reducing the dependence on external perturbing effects that might shift the beam tune at the beginning of the resonance crossing process.

The presented results suggest that the best strategy to improve the MTE efficiency is to excite the particles at a frequency that follows the changing horizontal tune along the cycle.
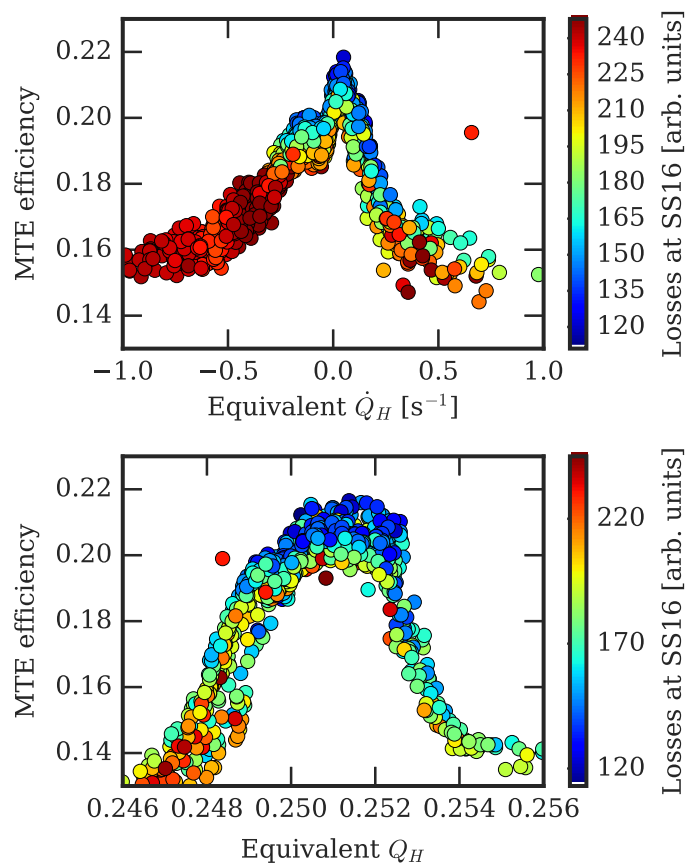

FIG. 15. Dependence of $\eta_{\mathrm{MTE}}$ on the slope (top) and initial value (bottom) of the excitation frequency generated by the transverse damper. The color scale provides the information on the extraction losses as measured by the BLM in SS16.

\section{NONLINEAR OPTICS AT EXTRACTION}

Subsequent to the successful splitting in the horizontal phase space, the beamlets have to be rotated in order to correctly position them at SMH16. The results of the previously discussed time-dependent simulations revealed that the operationally used rotation process occurred very quickly (see also Fig. 10) and, therefore, nonadiabatically. Eventually, this results in detrapping, filamentation and emittance blow-up, as the particles captured inside the islands are not able to follow the rapid motion of the SFPs (see Fig. 16). This is unfavorable for the subsequent extraction process, as these particles are lost during the rise of the fast bump, which reduces the overall extraction efficiency and increases the activation of the extraction region.

Further investigations of the final rotation at the location of SMH16 were conducted with PTC. In Fig. 17 (top), simulation results of the horizontal phase space of the external island at SMH16 are illustrated during the time span of the rotation. It is worth mentioning that this process occurs due to a change in the octupolar configuration of the lattice, as the strengths of the dedicated MTE octupoles is reduced toward the end of the cycle. At a cycle time of
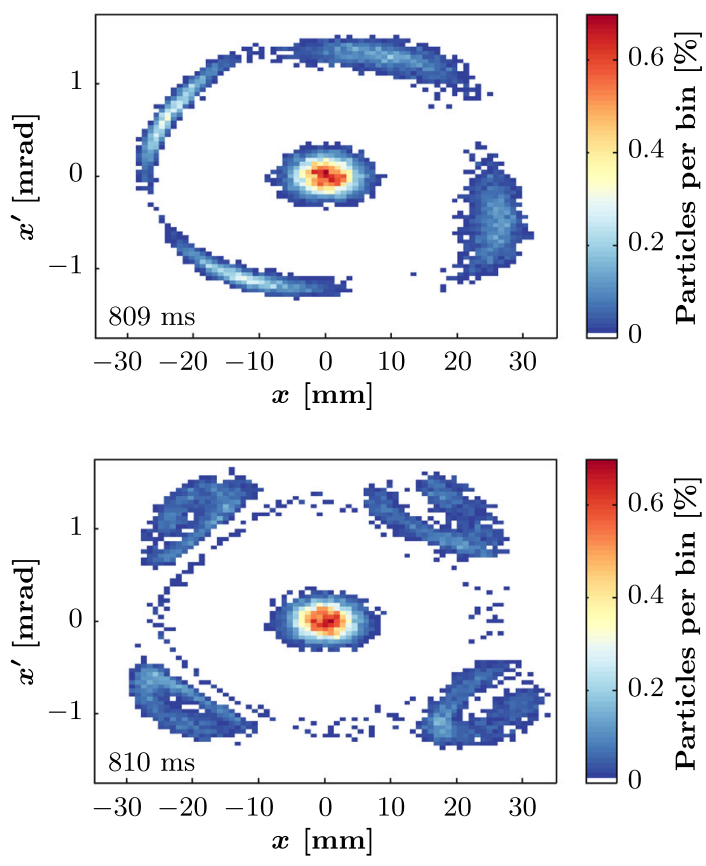

FIG. 16. Simulated horizontal phase space portraits in SS01 during a nonadiabatic final rotation. Once the beamlets are sufficiently separated (see Fig. 7), the rotation occurs within 2000 turns. The top figure shows an intermediate situation after 1000 turns and the phase space in the bottom corresponds to the end of the rotation process. Due to the nonadiabatic rotation detrapping has occurred, leading to particles captured between the core and the islands, and filamentation inside the islands is ongoing. The respective cycle times are indicated in the bottom left corners of the plots. 
$809 \mathrm{~ms}$, the beamlet is found to be perfectly positioned for extraction, as $x_{\mathrm{SMH} 16}^{\prime} \approx 0 \mathrm{mrad}$, and its size is significantly reduced compared to the configurations at the beginning and the end of the rotation. If the rotation occurred adiabatically and could be frozen at this instant, minimum extraction losses would be expected for such a configuration. This is based on the fact that for an adiabatic rotation, no particles remain in between the islands and the core and, furthermore, the clearance between the beam and the extraction septum is increased by reducing the beamlet's size.

Even though the islands' phase space topology is rather distorted, the $\beta_{x}$-function is a representative measure of the beam size in the close vicinity of the SFP. Its behavior for the external island is shown in Fig. 17 (bottom), and the rotation is found to be accompanied by a kind of discontinuity in the function.

The presented results led to the understanding that (i) the rotation has to occur significantly slower to preserve the splitting efficiency $\eta_{\mathrm{MTE}}$, and (ii) the surface of the islands can be reduced by properly choosing the multipolar configuration. Nevertheless, it has to be considered that any reduction of the surface is always accompanied by a
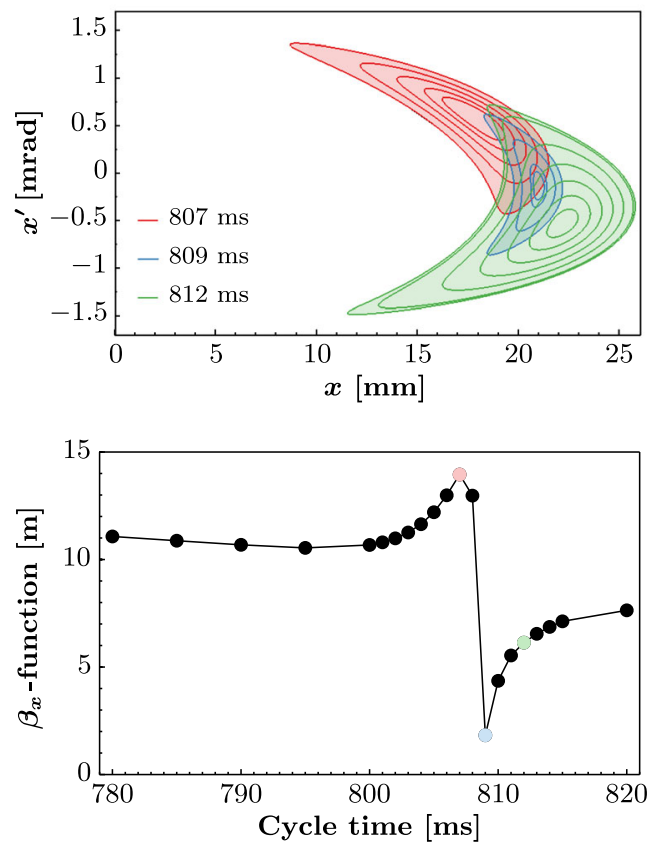

FIG. 17. Time-independent PTC simulation results to demonstrate the behavior of the external island at the entrance to SMH16 during the final rotation. Top: Horizontal phase space. As indicated by the time-dependent simulation results, the island is actually rotated and squeezed before it expands again. The legend indicates the different instances along the cycle. Bottom: Evolution of $\beta_{x}$-function. To reduce losses at SMH16, the optimum position of the external beamlet would correspond to the situation at $809 \mathrm{~ms}$. The colored markers correspond to the situations shown in the top figure. certain probability of detrapping [4]. This surface reduction in combination with the intended adiabatic rotation can eventually be achieved by acting either on the sextupolar or the octupolar component of the magnetic field. As acting on the octupolar component is accompanied by a significant change of the amplitude of the SFPs in the islands, it was decided to slowly adapt the sextupolar component to perform an appropriate rotation.

As a starting point the multipolar configuration at the end of the splitting process, i.e., before the final rotation takes place, was chosen. Subsequently, simulations were performed to investigate the susceptibility of the positions of the SFPs to changes of the settings of one dedicated MTE sextupole in SS55 (X55). In Fig. 18 (top), the response of the SFP at SMH16 to variations of the strength of X55, measured in terms of its electric current $I_{\mathrm{X} 55}$, is shown. To provide proper extraction conditions, $x_{\mathrm{SMH} 16}^{\prime} \approx 0 \mathrm{mrad}$ is sought for the outermost island, which can be achieved for a setting of $I_{\mathrm{X} 55}=160 \mathrm{~A}$. Regarding the evolution of the $\beta_{x}$-function (shown in the bottom of Fig. 18), a similarity to Fig. 17 (bottom) is observed: close to the optimum settings, $\beta_{x}$ experiences a significant jump, which can be exploited to reduce the island's size.

In order to achieve the required final separation of the beamlets while adiabatically rotating them, it was decided to slowly increase $I_{\mathrm{X} 55}$ and, at the same time, carefully adjust the horizontal tune. The corresponding time-dependent simulation results are presented in Fig. 19
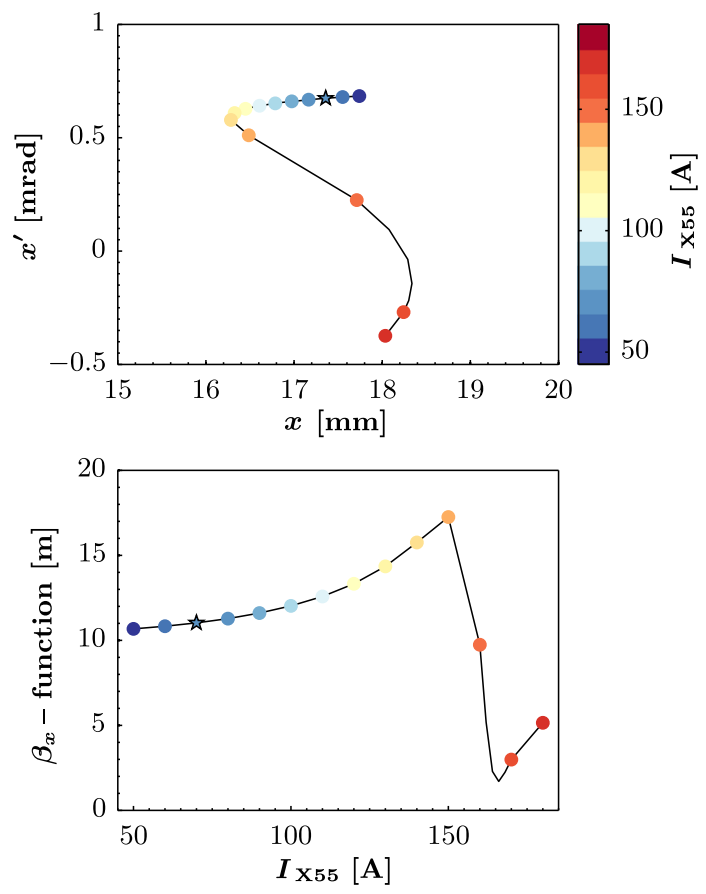

FIG. 18. Simulated dependency of the position of the SFP (top) and of the $\beta_{x}$-function (bottom) on the current of X55. In both cases the figures describe the situation for the external island at the location of SMH16. The initial values are indicated by the stars. 

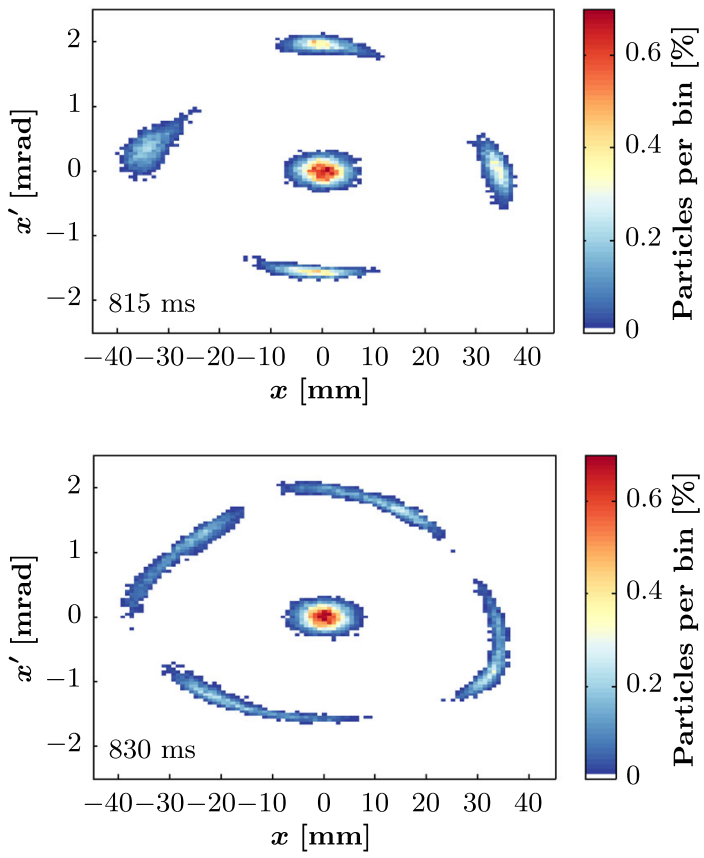

FIG. 19. Simulated horizontal phase space portraits in SS01 showing the evolution of an improved final rotation, which takes about six times longer than the one illustrated in Fig. 16. $\eta_{\mathrm{MTE}}$ is conserved during the process and the final surface of the islands, which is shown in the bottom plot, is significantly reduced. Note that the SFP of the external island is located at $x^{\prime} \approx-1 \mathrm{mrad}$, which is required to properly position the beamlet in SS15 and SS16. The respective cycle times are indicated in the bottom left corners of the plots.

and the islands are shown to slowly adapt to the new configuration. Thereby, they are elongated and the external one is reduced in horizontal size, which provides additional margin for the proper extraction process. With this configuration of the adiabatic rotation, the external beamlet (with $x>0$ ) can be properly positioned at TPS15 and SMH16. In Fig. 20, the horizontal phase space portraits at the corresponding locations are shown during the fast bump. The optimization of the phase space rotation allowed to obtain sufficient clearance between the septum blades and the beamlets.

Furthermore, this optimized configuration comes with the beneficial effect of significantly reducing the dispersion function of the external island at SMH16 from $D_{x}=$ $4.52 \mathrm{~m}$ to $D_{x}=0.11 \mathrm{~m}$. The beneficial impact of this improvement is also visualized by looking at Fig. 23.

The optimized multipole settings were experimentally implemented and a dedicated study showed that the extraction efficiency significantly increased from $93 \%$ to $97 \%$ due to the implementation of an adiabatic rotation.

However, it should be noted that the dedicated MTE sextupoles are applied for chromaticity correction. For the case under discussion, the horizontal chromaticity becomes actually slightly negative by increasing $I_{\mathrm{X} 55}$ to $160 \mathrm{~A}$,
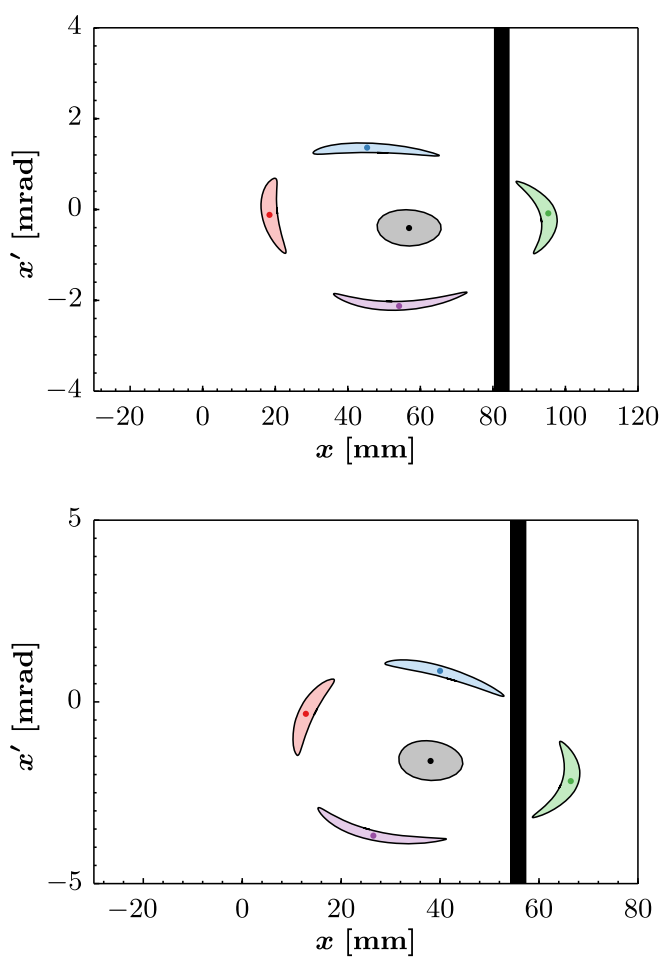

FIG. 20. Horizontal phase space portraits at TPS15 (top) and SMH16 (bottom) during the fast extraction bump. The shown surfaces of the islands represent single particle 2D tracking results, for a particle situated close to the separatrix. The optimization of the rotation has to consider the phase advance of $\approx 22.5^{\circ}$ between the two locations.

which is obviously undesired as MTE operation takes place above the transition energy. For the intensities, which are presently being operationally used, however, no transverse instabilities have been observed. On the one hand, this is based on the fact that the whole process of rotating the phase space takes place only $6 \mathrm{~ms}$ prior to debunching and $15 \mathrm{~ms}$ prior to extraction, which restricts the available time for an instability to develop. On the other hand, the intensity per beamlet is rather small due to the transverse splitting and the bunch length being rather long due to the reduced rf voltage.

The final step pursued to further increase the extraction efficiency was to correctly position the dummy septum with respect to SMH16, i.e., to establish shadowing conditions. The corresponding experimental results are set out in the following section.

\section{SEPTUM SHADOWING}

Based on the operational experience gathered with the MTE beam during 2010, the decision to install TPS15 was taken. More detailed information about its design and implementation can be found in the corresponding design report [6]. This device has been designed to absorb particles 
that would otherwise be lost at SMH16 during the rise time of the fast extraction kickers. These losses correspond to approximately $2 \%$ of the full beam intensity and arise due to the continuous longitudinal structure of the beam. It should be emphasized that the core and the islands are two disconnected phase space structures in the horizontal phase space, which need to jump the blades of the septa independently. Moreover, the rise time of the fast extraction kickers involved in the process is different.

TPS15 allows to reduce the radioactive activation in SS16 by relocating beam loss to the well-shielded SS15 and, therefore, the waiting time for any intervention in case of an issue with SMH16 is significantly reduced. Obviously, the opposite would be true for an intervention in SS15; however, a failure of the TPS15 would only affect the MTE beam, whereas SMH16 is required for the extraction of all proton beams. It is worth mentioning that a failure of TPS15 is unlikely due to its passive nature.

Even though the device is designated as septum, its purpose is purely passive as the interaction with the beam is only of mechanical nature and no magnetic field to deflect the beam is applied. The main component of this passive septum is a $400 \mathrm{~mm}$ long and $4.2 \mathrm{~mm}$ wide copper blade, whose angle and position can be precisely adjusted by means of two motors to shadow the magnetic septum (see Fig. 21).

SS15 was chosen as location for the dummy septum due to its proximity to SS16. It nevertheless has to be considered that the phase advance between those two sections of the accelerator is nonzero and the gap created downstream of TPS15 will have been slightly repopulated at the entrance of SMH16.

It is worth emphasizing that TPS15 constitutes an important aperture restriction in the extraction region. Therefore, the installation of TPS15 significantly complicated the MTE extraction process and impacted most of the

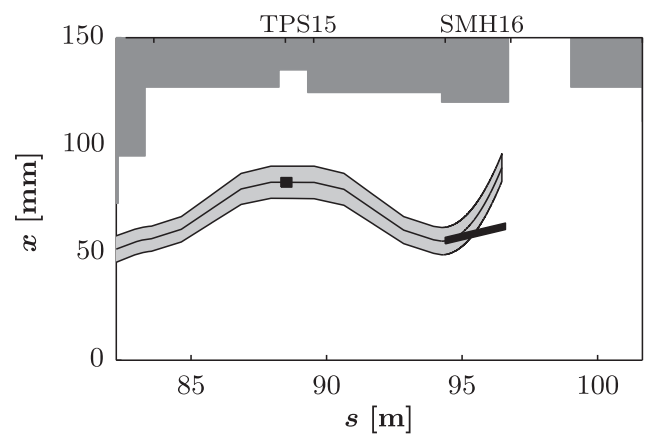

FIG. 21. During the rise time of the fast extraction kickers, the beam is swept from the internal to the external side of both TPS 15 and SMH16. Proper adjustment of the positions (and angles) of both septa allows to put SMH16 in the shadow of TPS15, and, therefore, reduce the losses in SS16. In the shown configuration the black rectangles indicate the septa at properly adjusted positions to provide shadowing. The efficiency of the shadowing might, however, be different between the core and the islands. other operational users as well by reducing the available aperture. Therefore, a redesign of the extraction trajectories was required for these users.

Prior to the activation of the fast extraction kickers a slow extraction bump with $6 \mathrm{~ms}$ rise time, which follows the shape of a quarter sine wave, is applied to approach both septa. During this period the particle motion is influenced by magnetic feed-down effects created by the nonlinear fields of the PFWs. This especially affects the positions of the islands, as the sextupolar and octupolar components of the PFWs generate a quadrupolar component by feeddown, while higher-order fields cause a minor change of the islands' size and shape. In the past, extraction bumps causing only a positive excursion of the beamlets' trajectories had been applied. Experimental experience showed undesirably high positive tune shifts, which caused the islands to be lost at SMH16, as they were transported to high amplitudes already before the maximum value of the slow bump was achieved. Therefore, a dynamic compensation of the tune during the rise time of the bump, which aimed at keeping the distance between the islands and the core constant, was concluded to be inevitable. However, additional studies revealed that such a compensation is extremely cumbersome to achieve and an alternative extraction bump was developed (see [10] for more detailed information). The main requirements for this bump were (i) to provide sufficient amplitude in SS15 to send the beam to the other side of TPS15 and (ii) to minimize the need for a tune compensation scheme during the rise time of the bump. Both points were achieved using a superposition of two $\pi$-bumps as shown in Fig. 22. The dedicated magnets are installed in SS12, 14, 20 and 22, and the depicted configuration allows to push the particle amplitude towards the outside of the machine at TPS15 without modifying the orbit at SMH16. However, an additional aperture restriction is created by the large negative excursion inside the MU19.

The major advantage of this new scheme is based on the alternation between positive and negative amplitudes

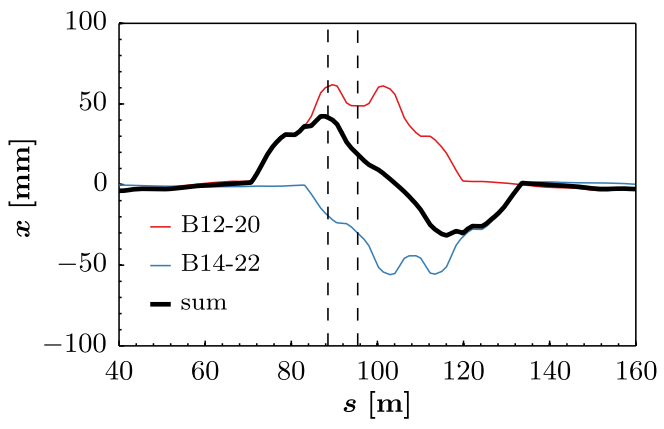

FIG. 22. Simulated horizontal orbit of a core particle at maximum amplitude of the slow extraction bump (black). This orbit is achieved as superposition of the red and blue curves, which represent the two $\pi$-bumps created by the dedicated magnets in SS12 and 20, and SS14 and 22, respectively. The locations of TPS15 and SMH16 are indicated by the dashed lines. 


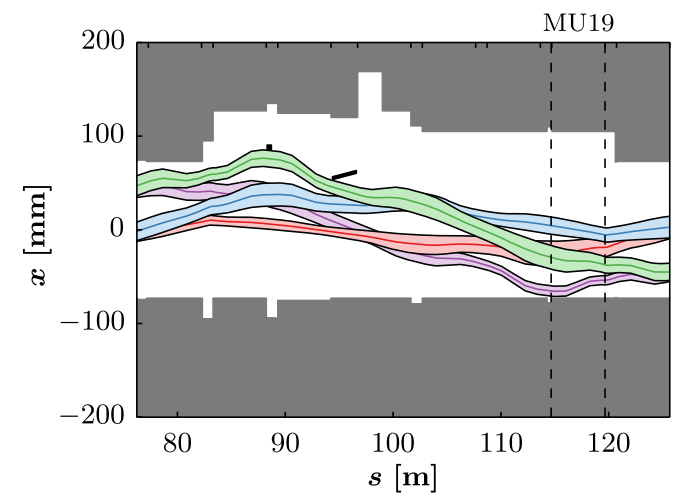

FIG. 23. Simulated horizontal orbits of the islands with the amplitude of the slow bump being at its maximum. The envelopes represent a beam size with extension of $3 \sigma_{x}$ and $2 \sigma_{s}$, based on Gaussian distributions in both planes $\left(\varepsilon_{x(1 \sigma)}^{n}=5 \mathrm{~mm} \mathrm{mrad}\right.$, $\left.\Delta p / p_{(1 \sigma)}=5 \times 10^{-4}\right)$. The horizontal mechanical aperture is represented in gray. The two septa are closely approached and the actual aperture limitation is caused by the proximity of the beam to the vacuum chamber inside MU19.

experienced by the beam: the resulting tune shift due to feed-down effects is almost self-compensated and only a minor tune correction is required to account for this effect. In order to visualize the extremely tight space constraints during the extraction process, the trajectories of the islands and the appropriate envelopes representing their beam sizes are displayed in Fig. 23. Once the slow bump has reached its maximum the outermost island is positioned closely to the septa and the fast extraction kickers are subsequently fired to extract the islands and the core over five turns. Simulation studies and experimental experience showed that TPS15 had to be moved to its minimum position of $80.5 \mathrm{~mm}$ and an angle of $0 \mathrm{mrad}$ to reduce beam loss during the fast bump by providing sufficient separation between the beam and the septum blade.

The successful modification of the slow bump allowed to determine the optimum shadowing configuration to minimize beam loss in SS16 and, therefore, a fine scan of the remaining degrees of freedom, namely the position and angle of SMH16, was experimentally conducted.

The essential component of the applied measurement procedure is the fast beam loss monitor (BLM) in SS16, which allows us to resolve beam loss on a time scale much shorter than one turn. Hence, losses occurring during the extraction of the islands can be distinguished from those created during the final turn. In Fig. 24 (top) a typical beam loss pattern during the last five turns, corresponding to $10.5 \mu \mathrm{s}$, is shown. The first peak is proportional to losses occurring during the rise time of the kickers to extract the islands (360 ns between 10-90\% of the maximum strength), while the second, smaller, peak describes beam loss caused by the rise of kicker for the core (70 ns between 10-90\% of the maximum strength) [6]. To evaluate the shadowing efficiency, the sum of the amplitudes of both peaks was
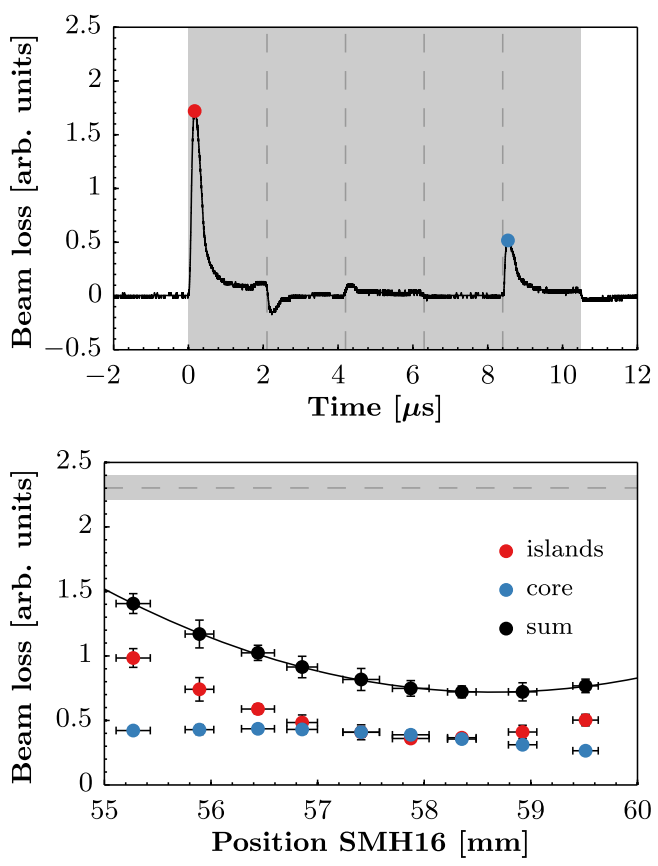

FIG. 24. Top: Signal of the fast BLM16. The grey area indicates a duration of five turns, with the dashed lines being separated by one turn. The red and the blue circles correspond to the maximum beam loss occurring during the extraction of the islands and the core, respectively. Bottom: Dependency of beam loss on the position of SMH16 for an angle of 0 mrad. Red and blue circles correspond to the losses of the islands and the core, respectively, and the black circles represent their sum. Error bars describe the standard deviation obtained over 10 consecutive measurements. The dashed line and the grey band indicate mean value and standard deviation of losses for the nominal septa settings.

considered as a figure of merit and the dependency of this value on the position and angle of SMH16 was investigated.

For an angle of $0 \mathrm{mrad}$, beam loss was observed to be a quadratic function of the position, with a minimum located at $58.65 \mathrm{~mm}$ (see Fig. 24 bottom). The losses measured at this position corresponded to a reduction by about a factor three compared to the value obtained for the settings of TPS15 and SMH16, which were considered operational at the time these measurements were conducted $(82.5 \mathrm{~mm} / 0 \mathrm{mrad}$ and $55.5 \mathrm{~mm} / 3 \mathrm{mrad}$, respectively). Based on these results, the following settings were chosen as new operational configuration: TPS15 at $80.5 \mathrm{~mm} / 0 \mathrm{mrad}$ and SMH16 at $57.5 \mathrm{~mm} / 1 \mathrm{mrad}$. These values constitute an acceptable compromise for both MTE and the other operational users. Measurements with the slow BLMs, which integrate beam loss over $1 \mathrm{~ms}$, are shown in Fig. 25 and confirm the intended reduction of losses in SS16. BLM15 was found to be the only remaining saturating device.

Regarding the extraction efficiency, an increase to $98 \%$ was observed on average, which has to be compared to $97 \%$ after the optimization of the nonlinear extraction optics 


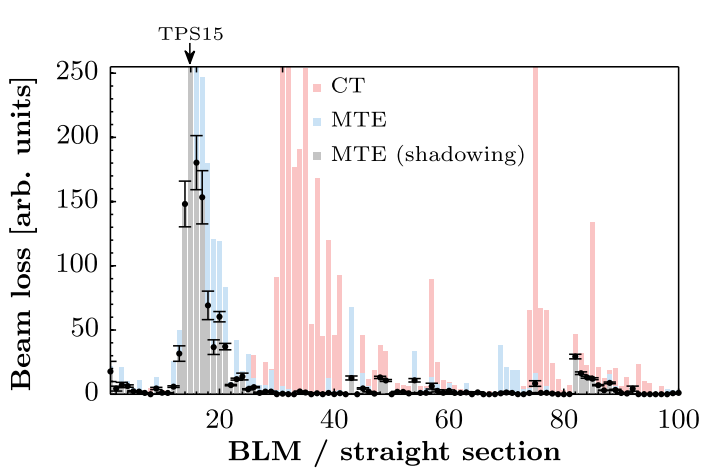

FIG. 25. Integrated beam loss measured after extraction on the MTE cycle using TPS15 and SMH16 in shadowing configuration. Only BLM15 is saturated, and beam loss at the adjacent SMH16 is significantly reduced. For comparison, the MTE case without TPS15 and the CT case are shown. Error bars for the case of MTE with shadowing correspond to the standard deviation of 500 consecutive measurements.

(see Sec. IV). In this context an interesting observation was made: on a cycle-by-cycle basis the beam losses in SS16 showed significant fluctuations. Positioning SMH16 in the shadow of TPS15 was a prerequisite for observing this phenomenon, as this led to a nonsaturated signal of the slow BLM16. For further investigations the variation of the frequency component of the main magnetic field at $5 \mathrm{kHz}$ was evaluated and compared to the measured signal of BLM16. In Fig. 26 both signals are shown to oscillate in a correlated manner, which led to the conclusion that the noise of the PCs of the PFWs still affected the MTE process, even though the ripple amplitude had been significantly reduced (see Sec. II). The effect is actually twofold: (i) the splitting process itself is affected, causing particles to be expelled from the islands and (ii) the islands' positions are modulated. Both effects contribute to the observed cycle-by-cycle variation of the losses in SS16 and, hence, the extraction efficiency and the reproducibility of the extraction trajectories in the transfer line toward the SPS.

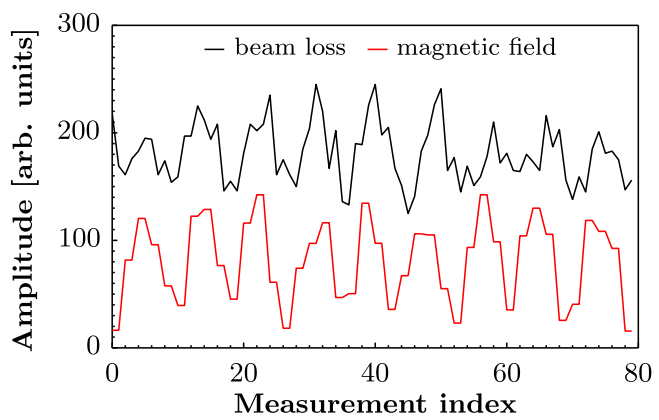

FIG. 26. Correlation between the signal of the slow BLM16 and the amplitude variation of the $5 \mathrm{kHz}$ component of the main magnetic field using TPS15 and SMH16 in shadowing configuration.

\section{CONCLUSIONS}

The efforts dedicated to the study and the implementation of the MTE technique in the PS have been rewarded by its first use in routine operation, which started in the second half of the 2015 SPS physics run. In this paper the key features, which are nonstandard topics in accelerator physics, of this extraction mode have been presented and discussed in detail.

Detailed measurements and simulation studies indicated clearly the strong and harmful impact of the lack of magnetic stability of the various accelerator elements. In particular, ripple effects generated by the power converters controlling the special coils installed in the PS main magnets have been proven to be the main source of the time-variation of the MTE efficiency.

Ideally, the frequencies of all PCs operating in switchmode should be in the order of $10 \mathrm{kHz}$ to reduce any interplay with the natural secondary frequencies of particles inside the islands. However, due to the load represented by certain circuits, this is technically not always feasible. Therefore, the feasibility of synchronizing the clocks controlling the switching of the different PCs has been investigated and will be implemented in 2017. With this improvement, the splitting process will still be affected, but it will no longer be subject to time-dependent fluctuations.

Controlling the secondary frequencies in order to keeping them small during the whole splitting process could be imagined. Thus, the overlap of the beam spectrum with that of the PCs would be reduced. However, as the secondary frequencies depend on the size of the islands and their distance from the center [19], this type of advanced splitting control requires a detailed investigation and optimization of the configuration of the non-linear elements.

Lastly, the use of the transverse damper has enabled increasing $\eta_{\mathrm{MTE}}$ from $0.14-0.15$ without excitation, to beyond 0.2 (up to $0.21-0.22$ ) with excitation. The impact of the horizontal dipolar excitation on the MTE efficiency has been studied in detail by means of scanning several of the available parameters. This has provided important information to guide further optimization of the excitation parameters and revealed that a high value of $\eta_{\mathrm{MTE}}$ is correlated with low extraction losses. The new feature enabling the possibility to generate a time-dependent excitation frequency has been probed and, based on its positive impact on the MTE efficiency, will be used in the next operational run. It is also worth stressing that the understanding of the impact of the transverse damper on beam splitting is the current topic of theoretical investigations.

The multitude of presented studies concerning the key features of the MTE extraction allowed to stabilize and increase $\eta_{\mathrm{MTE}}$ and the extraction efficiency. The latter experienced an improvement from 93\% to $97 \%$ due to the implementation of an adiabatic rotation of the beamlets in the horizontal phase space prior to extraction. A further increase of the extraction efficiency to above $98 \%$ was achieved by 
establishing a shadowing configuration between the newly installed TPS15 and the magnetic extraction septum SMH16.

Since LS1, the MTE commissioning has focussed on the establishment of reproducible experimental conditions and on rendering MTE operational. After having achieved this milestone, the optimization and simplification of the currently operationally used settings of the nonlinear MTE elements (see Fig. 2) can now be addressed.

In order to prepare the MTE scheme for potential future high-intensity beams, additional ideas, which require dedicated studies, will also be pursued. This concerns, on the one hand, the implementation of a longitudinal barrier bucket to provide a gap for the rise time of the fast extraction kickers and, on the other hand, investigations of the feasibility to extract at higher energy to alleviate the constraints in terms of limited available aperture.

\section{ACKNOWLEDGMENTS}

We would like to thank the PS Operations crew for the support during the commissioning stages.

[1] C. Bovet, D. Fiander, L. Henny, A. Krusche, and G. Plass, The fast shaving ejection for beam transfer from the CPS to the CERN $300 \mathrm{GeV}$ machine, IEEE Trans. Nucl. Sci. 20, 438 (1973).

[2] J. Barranco García and S. Gilardoni, Simulation and optimization of beam losses during continuous transfer extraction at the CERN Proton Synchrotron, Phys. Rev. ST Accel. Beams 14, 030101 (2011).

[3] R. Cappi and M. Giovannozzi, Novel Method for Multiturn Extraction: Trapping Charged Particles in Islands of Phase Space, Phys. Rev. Lett. 88, 104801 (2002).

[4] M. Giovannozzi, Report No. CERN-2006-011, 2006.

[5] A. Bazzani, C. Frye, M. Giovannozzi, and C. Hernalsteens, Analysis of adiabatic trapping for quasi-integrable areapreserving maps, Phys. Rev. E 89, 042915 (2014).

[6] C. Bertone et al., Report No. CERN-ACC-2014-0043, 2014.

[7] S. Gilardoni and D. Manglunki, Report No. CERN-2011004, 2011.

[8] J. Borburgh, S. Damjanovic, S. Gilardoni, M. Giovannozzi, C. Hernalsteens, M. Hourican, A. Huschauer, K. Kahle,
G. Le Godec, O. Michels, and G. Sterbini, First implementation of transversely split proton beams in the CERN Proton Synchrotron for the fixed-target physics programme, Europhys. Lett. 113, 34001 (2016).

[9] S. Abernethy et al., Operational performance of the CERN injector complex with transversely split beams, Phys. Rev. Accel. Beams 20, 014001 (2017).

[10] A. Huschauer, Ph.D. thesis, Vienna University of Technology, 2016.

[11] M. Buzio, A. Beaumont, P. Galbraith, G. Golluccio, D. Giloteaux, S. Gilardoni, C. Petrone, and L. Walckiers, Development of Upgraded Magnetic Instrumentation for CERN Real-Time Reference Field Measurement Systems, in Proceedings of the 1st International Particle Accelerator Conferenc IPAC-2010, Kyoto, Japan (JACoW, Kyoto, Japan, 2010), p. 310.

[12] P. Freyermuth, D. Cotte, M. Delrieux, H. Genoud, S. Gilardoni, K. Hanke, O. Hans, S. Mataguez, G. Metral, F. Peters, R. Steerenberg, and B. Vandorpe, CERN Proton Synchrotron Working Point Matrix for Extended Pole Face Winding Powering Scheme, in Proceedings of the 1st International Particle Accelerator Conferenc IPAC-2010, Kyoto, Japan (JACoW, Kyoto, Japan, 2010), p. 4551.

[13] O. Michels (private communication).

[14] F. Schmidt, E. Forest, and E. McIntosh, Reports No. CERN-SL-2002-044-AP, No. KEK-REPORT-20023, 2002.

[15] A. P. Shishlo, T. V. Gorlov, and J. A. Holmes, The Python Shell for the ORBIT Code, in Proceedings of the 10th International Computational Accelerator Physics conference, ICAP-2009, San Francisco, USA (JACoW, San Francisco, USA, 2009), p. 351.

[16] S. Hancock, M. Lindroos, E. McIntosh, and M. Metcalf, Tomographic measurements of longitudinal phase space density, Comput. Phys. Commun. 118, 61 (1999).

[17] A. Bazzani, F. Capoani, and M. Giovannozzi (to be published).

[18] A. Blas, S. Gilardoni, and G. Sterbini, Beam Tests and Plans for the CERN PS Transverse Damper System, in Proceedings of the 4th International Particle Accelerator Conference, IPAC-2013, Shanghai, China (JACoW, Shanghai, China, 2013), p. 2947.

[19] A. Bazzani, G. Servizi, E. Todesco, and G. Turchetti, Report No. CERN-94-02, 1994. 